\title{
Prognostic Value of the Future Liver Remnant Function Before Extensive Liver Resection in a Child with an Extremely Low Future Liver Remnant Volume
}

\author{
Akhaladze DG*, Rabaev GS, Merkulov NN, Tverdov IV and Grachev NS \\ Dmitry Rogachev National Medical Research Center of Pediatric Hematology, \\ Oncology and Immunology Ministry of Health of Russian Federation, Moscow, \\ Russian Federation \\ *Corresponding Author: Akhaladze DG, Dmitry Rogachev National Medical \\ Research Center of Pediatric Hematology, Oncology and Immunology Ministry of \\ Health of Russian Federation, Moscow, Russian Federation.
}

Received: June 10, 2021

Published: July 13, 2021

(C) All rights are reserved by Akhaladze DG., et al.

\begin{abstract}
Two-staged liver resections with portal vein embolization or associating liver partition and portal vein ligation for staged hepatectomy (ALPPS) are proven as an alternative to liver transplantation in adult patients. The widespread application of two-staged hepatectomies is associated with a high incidence of posthepatectomy liver failure (PHLF) after major liver resections. Nevertheless, two-stage resections are also characterized by a large number of complications, including PHLF due to the insufficient regeneration time or on the contrary the disease progression due to increased time between procedure stages. Insufficient values of the future liver remnant volume (FLR-V) or future liver remnant function (FLR-F) are the main limitations for single-stage hepatectomy. Wherein the future liver remnant function assessment has a greater sensitivity among adult patients. The lack of the data about the PHLF in pediatric patients and application of the FLR-V with a minimal borderline $25 \%$ as indications for two-stage liver resections, as well as the lower incidence of chronic concomitant diseases, suggest a greater significance of the liver functional capacity in children and the possibility to perform major liver resections in cases when FLR-V is less than allowable but FLR-F is sufficient. This is the first report describes how much FLR-V should be necessary in pediatric patients and confirms the possibility to wider perform one-stage liver resections in pediatric oncology without the risk of PHLF depending on the future liver remnant function assessment.
\end{abstract}

Keywords: Posthepatectomy Liver Failure; ALPPS; Hepatobiliary Scintigraphy; Future Liver Remnant Function; Future Liver Remnant Volume; Children

\section{Abbreviations}

ALPPS: Associating Liver Partition and Portal Vein Ligation for Staged Hepatectomy; CT: Computed Tomography; FLR-F: Future Liver Remnant Function; FLR-V: Future Liver Remnant Volume; INR: International Normalized Ratio; ISGLS: International Study Group of Liver Surgery; PHLF: Posthepatectomy Liver Failure; POD: Postoperative Day; PRETEXT: Pretreatment Extent of Disease

\section{Introduction}

The increased incidence of post-hepatectomy liver failure after major hepatectomy over the past two decades has led to development of two-stage liver resection techniques with portal vein embolization or associating liver partition and portal vein ligation for staged hepatectomy (ALPPS) [1]. The widespread advancement of these procedures was followed by high number of complications, including PHLF, and required to search new criteria which would

Citation: Akhaladze DG., et al. "Prognostic Value of the Future Liver Remnant Function Before Extensive Liver Resection in a Child with an Extremely Low Future Liver Remnant Volume". Acta Scientific Cancer Biology 5.8 (2021): 27-31. 
allow to assess the PHLF risk degree before surgery [2]. One of these criteria is future liver remnant volume (FLR-V) with a minimum threshold 25 - 40\% of the healthy liver parenchyma volume $[3,4]$. This wide range is explained with differences in the clinical status of patients and in the liver parenchyma functional condition $[2,4]$. These differences have led to more frequent application of FLR-F, for PHLF risk evaluation among adult patients [5]. On the other hand, there are no references about PHLF and FLR-F measurement in pediatric oncology, and the allowable FLR-V value $(25 \%)$ is the main indication for single- or two-staged hepatectomy. The lower incidence of concomitant diseases in childhood let us to suggest the possibility to perform the one-stage major hepatectomy in pediatric patients with FLR-V less than 25\%, in case of sufficient FLR-F value.

This report describes the case of one-stage extended right hepatectomy in a child, suffering from a malignant liver tumor, with critically low FLR-V.

\section{Case Presentation}

An ultrasound examination revealed a huge neoplasm of the right liver lobe in a 3 years old boy. The patient had no clinical signs of disease but was admitted to the Dmitry Rogachev National Medical Research Center of Pediatric Hematology, Oncology and Immunology. The diagnosis "Embryonic histological type hepatoblastoma of 1, 4, 6, 7, 8 hepatic segments (PRETEXT III), C1E0F0HOMONOPOV0" was established.

Neoadjuvant polychemotherapy was performed according to the SIOPEL-4 protocol for high-risk patients. During the control exam an alpha-fetoprotein decreased from $196000 \mathrm{ng} / \mathrm{ml}$ to 392 $\mathrm{ng} / \mathrm{ml}$, plus the tumor volume reduction from $175 \mathrm{ml}$ to $52 \mathrm{ml}$ was noted (Figure 1). The future liver remnant volume by CT volumetry $\left(\right.$ AUTOPLAN $^{\odot}$ ) after neoajuvant treatment came to $16.5 \%$. According to hepatobiliary scintigraphy with ${ }^{99 \mathrm{~m}} \mathrm{Tc}-$ mebrofenin the future liver remnant function was $3,95 \% / \mathrm{min} / \mathrm{m}^{2}$. Taking into the consideration the allowable FLR-F, necessity to keep specific therapy timing, the patient underwent one-stage extended right hepatectomy, segmentectomy 1 (Figure 2), ignoring the insufficient volume of the future liver remnant $(\mathrm{S} 2,3)$.

Histological examination confirmed the diagnosis hepatoblastoma with therapeutically induced $2^{\text {nd }}$ degree pathomorphosis. The resection margins contained no atypical cells.

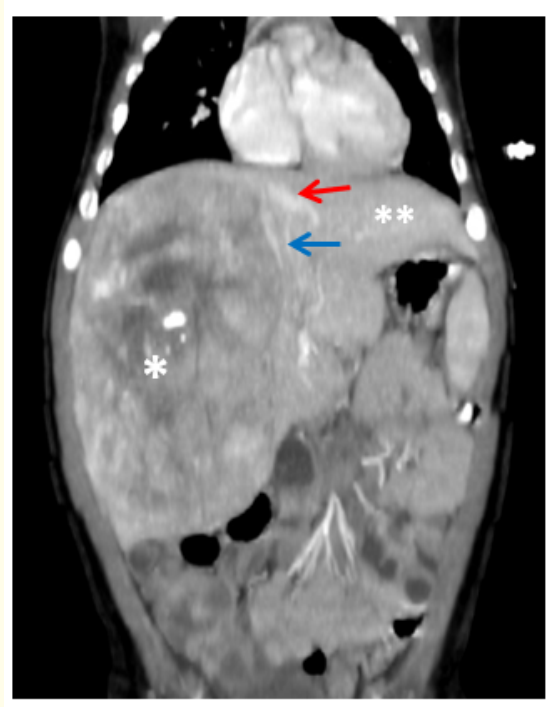

Figure 1: Preoperative CT scan. The tumor (*) involves 4,6,7,8,1 hepatic segments and infiltrates middle hepatic vein (blue arrow). Left lateral section ( ${ }^{* *}$ ). Left hepatic vein (red arrow).

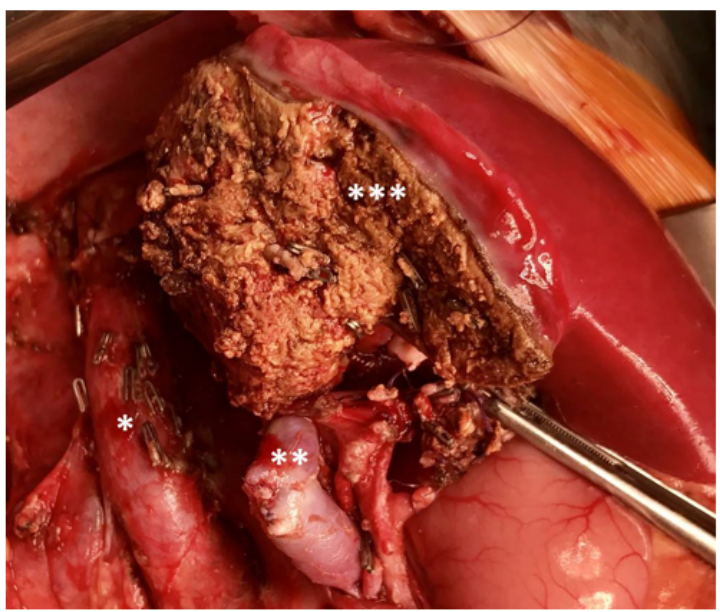

Figure 2: Extended right hepatectomy. The short veins draining the posterior segments and S1 (*) are clipped. Stump of the right portal vein branch ${ }^{* *}$ ). Resection margin S2.3 (***). 
No clinical signs of post-hepatectomy liver failure were noted in postoperative period. On the $5^{\text {th }}$ day, the serum bilirubin came to $29,3 \mu \mathrm{mol} / \mathrm{l}$, prothrombin was $40 \%$, the international normalized ratio (INR) - 2 (Figure 3-5). All that made possible to state the absence of liver failure when assessed using the 50/50 criteria" [6] or mild degree of PHLF (grade A according to ISGLS criteria [7]) without clinical manifestations (Table 1).

On $14^{\text {th }}$ postoperative day, patient continued the specific therapy in the Clinical Oncology department. After a follow-up examination, patient was discharged with complete remission.

At the time of the report writing, the follow-up period came to 20 months. There are no signs of main disease recurrence or liver failure.

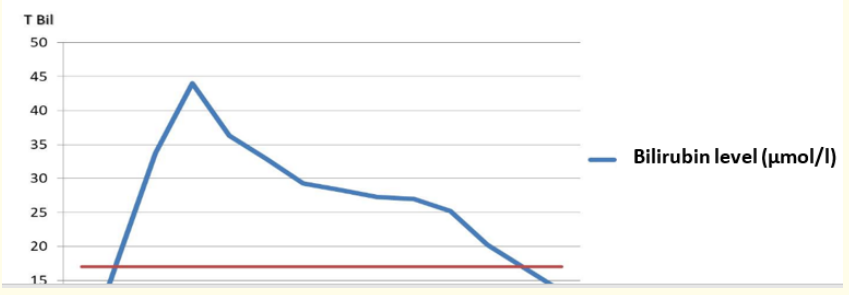

Figure 3: Diagram 1. Dynamics of the total bilirubin level in the postoperative period.

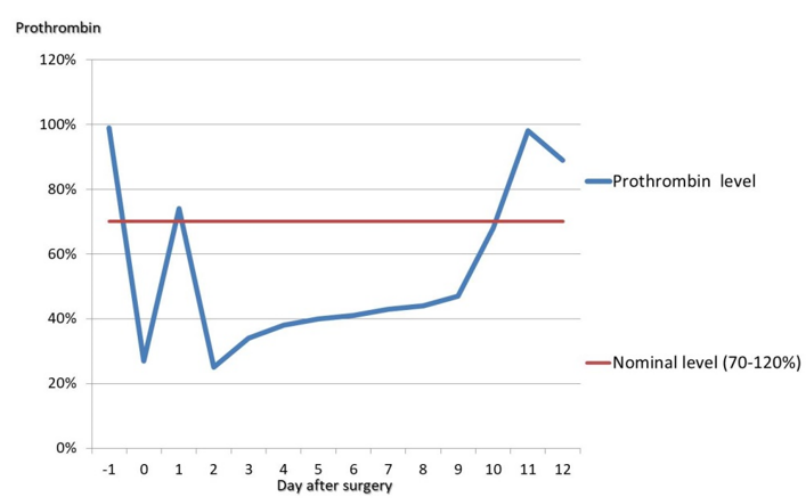

Figure 4: Diagram 2. Dynamics of the prothrombin level.

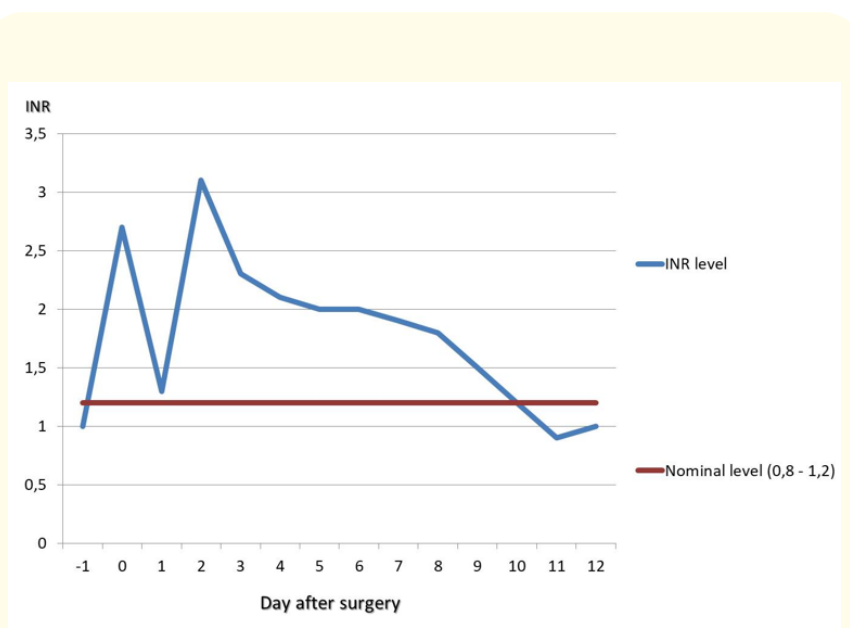

Figure 5: Diagram 3. INR level.

\begin{tabular}{|l|c|c|}
\hline \multicolumn{1}{|c|}{ Sign } & $\begin{array}{c}\text { Value in PHLF } \\
\text { degree A }\end{array}$ & Value in patient \\
\hline Specific therapy & Not required & Not required \\
\hline INR on $5^{\text {th }}$ POD & $<1,5$ & 2 \\
\hline Urine output & $>1,5 \mathrm{ml} / \mathrm{kg} / \mathrm{hour}$ & $3 \mathrm{ml} / \mathrm{kg} / \mathrm{hour}$ \\
\hline Creatinine & $<1500 \mathrm{mg} / \mathrm{l}$ & $192 \mathrm{mg} / \mathrm{l}$ \\
\hline $\mathrm{O}_{2}$ saturation & $>90 \%$ & $98 \%$ \\
\hline
\end{tabular}

Table 1: Indicators of liver failure in the postoperative period.

\section{Discussion and Conclusion}

Post-hepatectomy liver failure is the main cause of death among postoperative complications after liver resection, with incidence coming to $1,2-32 \%[2,4]$. Significant difference between the incidences of the PHLF is explained by absence of an unitary definition for this complication for a long time. In 2011 Rahbari., et al. [7] proposed serum bilirubin level and international normalized ratio (INR) as two main assessment criteria for this condition. So PHLF is diagnosed in case of increased serum bilirubin or INR for more than 5 days after surgery. After establishment of a unitary term for post-hepatectomy liver failure was followed by increased complication incidence over the past decades due to the wider application of extensive liver resections [3,4]. Estimation of the future liver remnant volume before surgery can reduce the incidence of PHLF. 
Relative availability and low cost computed tomography brought this technique widespread implementation, including in pediatric oncology [8]. If the FLR-V value is below the allowable level, to twostage resection using portal vein embolization, portal vein ligation, or ALPPS technique are preferred [2,9]. ALPPS allows to achieve the fastest future liver remnant hypertrophy in comparison with other types of two-stage resections. However, according to the literature considering adult patients, Matsuo., et al. [10] reported 7 - 14 days period between the liver resection stages as insufficient for "maturation" and functional regeneration of hepatocytes, despite a volumetric increase of future liver remnant. Liver failure with incidence up to $17 \%$, large number of deaths (up to $12 \%$ ) and high level of complications reaching 83\% after ALPPS, also lead to increase of the time between stages of surgery breaking down specific therapy timing and the increasing risk of the main disease progression [11].

The minimum cut-off values for FLR-V are $25-40 \%$ of the healthy liver parenchyma volume [3]. The differences in this numbers can be explained by higher risk of PHLF in patients with cirrhotic liver, portal hypertension, decreased hepatocyte function after cholangitis, neoadjuvant treatment with hepatotoxic drugs, as well as concomitant chronic diseases in the elderly [12]. So, volumetry does not reflect the real condition of the liver parenchyma, in comparison with the functional tests. In some cases, FLR-V assessment leads to patients wrong stratification into the two-stage liver resections or transplantation groups and this mistake may increase the risk of severe complications [13].

The hepatocytes function is composed by processes including biological substances capture, biotransformation, synthesis and excretion. Up to date, there are no diagnostic methods that allow to estimate all these processes in total, that explains the large number of different methods for assessment of the liver parenchyma functional reserve and lack of a gold standard method. Hepatobiliary scintigraphy with ${ }^{99 \mathrm{~m}} \mathrm{Tc}$-mebrofenin reflects incretory and excretory function of hepatocytes, makes it possible to determine the functional activity of the parenchyma in liver segments separately [14]. This advantage has brought widespread for FLR-F evaluation before surgery using hepatobiliary scintigraphy [15-17].

In according to literature dedicated to pediatric oncology, there are no references mentioning posthepatectomy liver failure, as well as methods for assessing the functional reserve of the future liver remnant in children. Hepatobiliary scintigraphy has been used in pediatric population since last century for limited indications, usually including congenital or acquired diseases of the biliary tract. Whereas CT volumetry remains the basic study for preventing PHLF in pediatric oncology, and the FLR-V minimal value $(25 \%)$ was extrapolated from adult patients.

The absence of literary data concerning PHLF in children is most likely associated with a lower incidence of liver tumors in children compared with adults. Nevertheless, the most frequent malignant liver tumor in children remains hepatoblastoma, this neoplasm as embryonic tumor, characterized by a rapid growth rate and often requires an extended or maximum permissible liver resection and explains attempts to perform two-stage resections, including ALPPS in children since 2014 [8]. To date, there are 7 publications devoted to the implementation of ALPPS in children, reporting 13 cases of this operation in total. The future liver remnant volume in these publications ranged from $14.7 \%$ to $39.3 \%$ with a median of $22.2 \%$, and time between stages was from 6 to 16 days (median 9 days). The following postoperative complications were described: infectious complications requiring therapy in the intensive care unit after the first stage, local relapse on the $30^{\text {th }}$ postoperative day with subsequent progression of the disease; hydrothorax, which required surgical treatment, dynamic bowel obstruction after the first stage, which required parenteral nutrition, mechanical ventilation after the first stage due to liver remnant intensive hypertrophy and lung compression, pneumonia after the second stage. There were no cases of post-hepatectomy liver failure.

In conclusion, we note that the lack of data about PHLF, the intensity of hepatocytes functional regeneration in comparison with adult patients requires the introduction of methods that assess future liver remnant function for exact indications for two-stage liver resections, especially ALPPS. This method, in some cases, can be an alternative to liver transplantation, in other cases, like onestage resection, ALPPS increases risk of PHLF. We can assume that patients with acceptable FLR-F values do not need two-stage liver resection, and one-stage extensive liver resection will be safe, despite the FLR-V value below the permissible level. This clinical case is the first report about successful extensive resection for a liver malignant neoplasm in a child with the extremely low volume of the healthy parenchyma $(16,5 \%)$. FLR-F evaluation makes possible to perform extensive liver resection with minimal risks of clinically 
significant PHLF development, even when the remnant volume is significantly below than threshold value.

\section{Conflict of Interest}

There is no conflict of interest.

\section{Bibliography}

1. Clavien PA., et al. "Strategies for Safer Liver Surgery and Partial Liver Transplantation". The New England Journal of Medicine 356.15 (2007): 1545-1559.

2. Van Den Broek M., et al. "Liver failure after partial hepatic resection: Definition, pathophysiology, risk factors and treatment". Liver International 28.6 (2008): 767-780.

3. Vauthey JN., et al. "Standardized measurement of the future liver remnant prior to extended liver resection: Methodology and clinical associations". Surgery 127.5 (2000): 512-519.

4. Kauffmann R and Fong Y. "Post-hepatectomy liver failure". Hepatobiliary Surgery and Nutrition 3.5 (2014): 238-246.

5. Olthof PB., et al. "99mTc-mebrofenin hepatobiliary scintigraphy predicts liver failure following major liver resection for perihilar cholangiocarcinoma". Hpb 19.10 (2017): 850-858.

6. Balzan S., et al. "The "50-50 criteria" on postoperative day 5: An accurate predictor of liver failure and death after hepatectomy". Annals of Surgery 242.6 (2005): 824-829.

7. Rahbari NN., et al. "Posthepatectomy liver failure: A definition and grading by the International Study Group of Liver Surgery (ISGLS)". Surgery 149.5 (2011): 713-724.

8. Chan A., et al. "Little girl who conquered the "ALPPS". World Journal of Gastroenterology 20.29 (2014): 10208-10211.

9. Ribero D., et al. "Preoperative cholangitis and future liver remnant volume determine the risk of liver failure in patients undergoing resection for hilar cholangiocarcinoma". Journal of the American College of Surgeons 223.1 (2016): 87-97.

10. Matsuo K., et al. "Histologic features after surgery associating liver partition and portal vein ligation for staged hepatectomy versus those after hepatectomy with portal vein embolization". Surgery 159.5 (2016): 1289-1298.
11. Sandstrom P., et al. "ALPPS Improves Resectability Compared with Conventional Two-stage Hepatectomy in Patients with Advanced Colorectal Liver Metastasis: Results from a Scandinavian Multicenter Randomized Controlled Trial (LIGRO Trial)". Annals of Surgery 267.5 (2018): 833-840.

12. Yokoyama Yu., et al. "Mechanism of impaired hepatic regeneration in cholestatic liver". Journal of Hepato-Biliary-Pancreatic Sciences 14 (2007): 159-166.

13. Chan A., et al. "ALPPS Versus Portal Vein Embolization for Hepatitis-related Hepatocellular Carcinoma". Annals of Surgery 20 (2019): 1-9.

14. de Graaf W., et al. "Nuclear imaging techniques for the assessment of hepatic function in liver surgery and transplantation". The Journal of Nuclear Medicine 51.5 (2010): 742-752.

15. Olthof PB., et al. "Hepatobiliary scintigraphy to evaluate liver function in associating liver partition and portal vein ligation for staged hepatectomy: Liver volume overestimates liver function". Surgery 162.4 (2017): 775-783.

16. Chapelle T., et al. "Future remnant liver function estimated by combining liver volumetry on magnetic resonance imaging with total liver function on $99 \mathrm{mTc}-$ mebrofenin hepatobiliary scintigraphy: Can this tool predict post-hepatectomy liver failure?" HPB 18.6 (2016): 494-503.

17. Rassam F., et al. "Quantitative assessment of liver function using hepatobiliary scintigraphy: The effect of microcirculatory alterations after portal vein embolization". Nuclear Medicine Communications 40.7 (2019): 720-726.

\section{Volume 5 Issue 8 August 2021 (C) All rights are reserved by Akhaladze DG., et al.}

